\title{
Modeling ALS/FTD in mice: Updates on the C9orf72 BAC transgenic mice
}

Mordes, D.A. et al Neuron 108, 775-783.e4 (2020)

Nguyen, L. et al. Neuron 108, 784-796.e3 (2020)

Amyotrophic lateral sclerosis (ALS) is a neurodegenerative disease characterized by a progressive loss of motor neurons, which leads to muscle weakness and eventual paralysis and respiratory failure. Mutations in the C9orf72 gene have been identified as the major cause of ALS, accounting for $40-50 \%$ of familial ALS cases and $~ 7 \%$ of sporadic cases. The mutations, which consist of hexanucleotide repeat expansions (GGGGCC) have also been linked to frontotemporal dementia (FTD).

The high prevalence of these mutations in ALS and FTD has led to the development of several mouse models, including several bacterial artificial chromosome (BAC) transgenic mice, harboring GGGGCC repeat expansions. Most BAC transgenic models have pathological features of ALS/FTD, including accumulation in the CNS of repeat RNAs and dipeptide repeat proteins created via repeat-associated non-AUG (RAN) translation of repeat RNA. However, to date, very few models such as C9-500 transgenic mice-a C9orf72 BAC mouse model with $\sim 500$ repeats developed by Liu et al. at the University of Floridahave shown reduced survival and signs of motor deficits and neurodegeneration. C9-500 transgenic mice could be valuable to study C9orf72 pathogenesis but a new paper calls for caution when interpreting the phenotype of these mice.

In Neuron, Mordes et al. publish a Matter Arising article as a response to the study by Liu et al. They report that two independent colonies of C9-500 transgenic mice phenotyped at Harvard University and Johns Hopkins University did not show abnormalities in survival, motor function or neurodegeneration. The team provides two possible explanations for the phenotypic differences between their study and the work by Liu. et al. "One explanation for the disparate nature of our findings relative

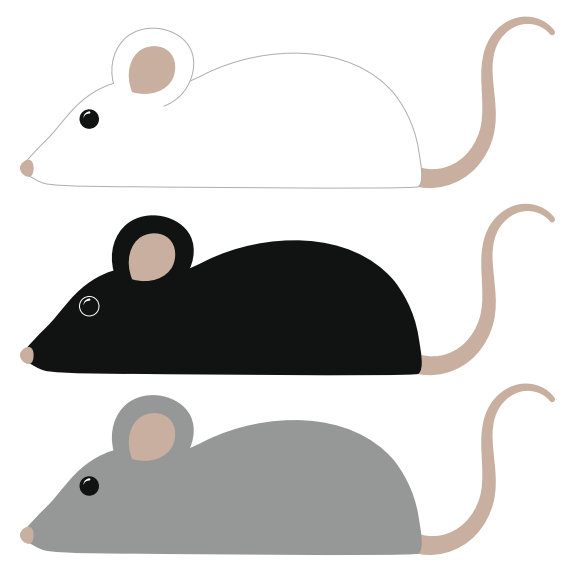

Credit: Marina Spence/ Springer Nature

to those reported by Liu and colleagues is that housing environment somehow contributes to phenotypic manifestations in C9-500 animals," write the investigators. This hypothesis is based on previous studies that have shown that vivarium-dependent commensal micro-organisms can affect the severity of symptoms in other mouse models of ALS.

The second explanation proposed by Mordes et al. is that the genetic background of the mice might have compromised the analysis. C9-500 transgenic animals have been generated and studied on a FVB/N genetic background, which has been associated with a syndrome of sudden death and variable CNS lesions named space cadet syndrome (SCS). Mordes et al. suggest that FVB C9-500 animals in the Liu et al. study might have developed more signs of SCS by chance than non-transgenic (NT) FVB mice.

In the same issue of Neuron, Nguyen et al. from the University of Florida reply to the Matter Arising article by emphasizing that the survival and motor phenotypes shown by Liu et al. could be reproduced in four different cohorts of FVB C9-500 $\mathrm{BAC}$ transgenic mice, including two new studies performed independently at the University of Bern and the University of Rochester Medical Center. In a previous report, Nguyen et al. had also shown that the phenotype of C9-500 mice including decreased survival, DigiGait abnormalities, open field abnormalities, motor neuron loss, RNA foci and RAN protein accumulation could be improved by targeting GA RAN proteins with a-GA1 antibody, which would further support that the FVB C9-500 phenotype is caused by RAN protein pathology and not a FVB-strain phenotype.

"Although occasional seizures occurred in NT FVB mice, as reported in the initial study by Liu et al. (2016), the presence of seizures did not interfere with detection of clear phenotypic differences in behavior, neuropathology, and survival between C9-BAC mice and their NT littermates," explain Nguyen et al. They propose that the small number of control NT animals used by Mordes et al. might explain the absence of significant differences in survival between C9-500 transgenic and NT littermates, and that methodological differences might account for weight loss, grip strength, and open field discrepancies between studies.

Further investigations are needed to identify the genetic and/or environmental factors responsible for the differences in phenotype observed in C9-BAC mice across laboratories. Determining whether the C9orf72 transgene is sufficient to trigger disease-associated phenotypes in mice is an important step towards our understanding of C9orf72 ALS/FTD pathogenesis.

Alexandra Le Bras

Published online: 18 December 2020 https://doi.org/10.1038/s41684-020-00693-9 